\title{
LA GUERRE DU « NÉNUFAR » N’A PAS EU LIEU EN BELGIQUE
}

\author{
Michèle Lenoble-Pinson \\ Conseil international de la langue française \\ France \\ Professeur émérite de l'Université Saint-Louis \\ Belgique \\ michele.lenoble@skynet.be
}

doi.org/10.15452/SR.2021.21.0002

Résumé. Peut-on toucher à l'orthographe sans nuire à la langue ? En France, au Québec, en Suisse romande et en Belgique francophone, les rectifications ont reçu l'aval d'organismes officiels, dont le Conseil international de la langue française (Paris). Les Belges sont habitués à la variation linguistique. Dans la presse, les échos favorables et défavorables se sont équilibrés. Des périodiques, les départements de langues romanes des universités, des enseignants, les Championnats d'orthographe ont adopté les rectifications. Un organisme indépendant, l'APARO (Association pour l'application des recommandations orthographiques), s'en est fait le diffuseur efficace et bénévole et a publié des livrets de référence. Depuis 1998, deux documents ministériels les recommandent. Manuels scolaires, dictionnaires, correcteurs orthographiques, sites de l'internet suivent ou signalent les recommandations. Elles s'implantent lentement mais sûrement en Belgique.

Néanmoins, certains s'interrogent encore. S’agit-il d'une révolution? Les graphies rectifiées gênent-elles la lecture ? Cohabitent-elles avec les anciennes ? Existe-t-il d'autres projets de rationalisation de l'orthographe? Oui.

Mots-clés. Nouvelle orthographe. Rectifications. Recommandations. Renouvo. Championnats d'orthographe de Belgique.

Abstract. The War of the "Water Lily" Did Not Take Place in Belgium. Can we modify orthography without harming the language? In France, in Quebec, in the Francophone parts of Switzerland and Belgium, the orthographic rectifications were approved by official organizations, among which International Council for the French Language, based in Paris. The Belgians are used to linguistic variation. Favourable and unfavourable echoes in the press are 
nowadays balanced. Periodicals, the university departments of the Romance languages, teachers and the Orthographic Championships accepted the rectifications. An independent organisation, l'APARO (Association for the Application of the Orthographic Recommendations), is spreading them efficiently and voluntarily and has published some reference books. Since 1998, two ministerial documents have recommended them. School textbooks, dictionaries, spelling checkers and Internet sites follow or point out these recommendations. They are establishing themselves slowly but surely in Belgium.

Nevertheless, some questions remain. Is it about a revolution? Does new orthography obstruct reading? Does it coexist with old orthography? Are there other projects streamlining orthography? Yes, there are.

Keywords. New orthography. Rectifications. Recommendations. Renouvo. Belgian Orthographic Championships. 
«Bientôt le cours de la Vivonne s'obstrue de plantes d'eau. Il y en a d'abord d'isolées comme tel nénufar à qui le courant au travers duquel il était placé d'une façon malheureuse laissait si peu de repos que, comme un bac actionné mécaniquement, il n'abordait une rive que pour retourner à celle d'où il était venu, refaisant éternellement la double traversée. »

Marcel Proust, $A$ la recherche du temps perdu.

La « guerre du nénufar » désigne, par dérision, les combats médiatiques, parfois virulents, menés contre les rectifications orthographiques, dont l'une propose en effet de renoncer au ph de nénuphar qui, pour certains, faisait le charme du mot. La suite ph est injustifiée dans nénuphar ainsi que dans raphia et typhon, dont le rapport du Conseil supérieur de la langue française ne parle pas (Goosse, 1991 : 83). Littré et Proust ont toujours écrit nénufar parce que le mot ne remonte pas au grec, mais à l'ancien persan nîlüfar, de même sens, par l'ancien arabe, où la graphie $p h$ n'existe pas.

\section{Introduction'}

Peut-on toucher à l'orthographe sans nuire à la langue?

Oui, parce que l'orthographe n'est pas la langue. Beaucoup les confondent. L'orthographe n'est que le vêtement de la langue. Or un vêtement vieillit, s'use et se démode. Toucher au vêtement qu'est l'orthographe et le modifier, ce n'est pas toucher à la langue. Que l'on écrive un mille-pattes ou un millepatte comme un millefeuille, on renvoie au même mot, avec la même signification. Même si les éditeurs ont modernisé les graphies de Montaigne, Molière et Chateaubriand, leurs textes restent écrits en français. Faire évoluer des graphies, en corriger certaines, rationaliser l'orthographe, ce n'est ni assassiner la langue, ni l'appauvrir, ni opérer un nivèlement par le bas puisque l'on ne touche pas à la langue, on ne touche qu'à son vêtement. Ces ajustements ne remettent point en cause l'attachement que les francophones portent à leur langue.

Puisque le français n'est pas une langue morte comme le latin, mais une langue vivante, il est normal que son usage évolue. La prononciation, le vocabulaire usuel, les terminologies scientifiques et techniques, la féminisation des noms de professions (Lenoble-Pinson, 2005) évoluent. Les réformes participent à cette évolution. Ainsi Les rectifications de l'orthographe, énoncées dans le rapport du Conseil supérieur de la langue française publié le 6 décembre 1990, dans les Documents administratifs du Journal officiel de la République française, montrent-elles une évolution de l'usage.

Ces rectifications, l'Académie française les a approuvées à l'unanimité. Elles ont également reçu l'aval d'instances officielles en France, au Québec, en Suisse romande et en Communauté française de Belgique, devenue la Fédération Wallonie-Bruxelles (Biedermann-Pasques ; Jejcic, 2006). Le Conseil international de la langue française (CILF, Paris) a suivi l'Académie en les introduisant dès 1991 dans toutes ses publications sans que personne n'y trouvât à redire. En 2020, les rectifications orthographiques ont trente ans ! Lâge de la maturité.

1 Ce texte applique les rectifications de 1990. 


\section{En Belgique}

En Belgique francophone, le Conseil supérieur de la langue française donna d'emblée un avis favorable aux rectifications et, depuis 1991, elles s'implantent lentement mais surement. Le Service de la langue française de la Fédération Wallonie-Bruxelles, qui les a adoptées dans ses textes, ses publications et sur son site, y contribue : www.languefrancaise.be. Les rectifications sont utilisées soit consciemment par des scripteurs qui les connaissent, soit inconsciemment par de plus en plus d'usagers qui ne les ont pas apprises mais qui les appliquent parce que la plupart correspondent à l'évolution de l'usage écrit du français. Ainsi, parmi les étudiants qui entrent à l'université, trois sur cinq mettent un point sur le $i$ de (il) parait, connait et maitrise. Une magistrate écrit spontanément charriot, déciller, deux-cents.

\subsection{L'orthographe est l'habit de la langue}

L'orthographe n'est pas la langue, elle n'est que son habit ou une technique pour l'écrire. Elle peut évoluer sans porter atteinte à son intégrité. Cette prise de conscience, qui a pour effet de rassurer les usagers inquiets devant le changement, accompagna l'entrée des rectifications dans le royaume de Belgique. De plus, celle-ci bénéficia du grand avantage d'être portée par monsieur André Goosse, professeur à l'Université catholique de Louvain, qui fut un témoin direct de l'établissement des rectifications. En effet, André Goosse fit partie du comité d'experts qui prépara les rectifications au sein du Conseil supérieur de la langue française institué par monsieur Michel Rocard. Monsieur Goosse fut un des principaux agents de la « réforme », voire «l'âme de la réforme ». Sa remarquable participation au Conseil supérieur et au comité d'experts a d'emblée disposé favorablement ses compatriotes.

Philologue et grammairien, André Goosse veilla constamment, avec érudition, à remettre les choses à leur place en répondant aux arguments des opposants et en replaçant le débat souvent passionné dans un contexte élargi, plus serein et parfaitement informé. De surcroit, il ne cessa de publier des articles en plus de son ouvrage sur La «nouvelle » orthographe (Goosse, 1991). Cette étude objective, à la fois synthétique et complète, accompagne les aménagements orthographiques de justifications et de commentaires éclairés sans exclure l'agrément ni l'humour. Successeur du grand grammairien belge Maurice Grevisse, André Goosse inclut les rectifications dans la $13^{e}$ édition (1993) du Bon usage qu'il préparait ainsi que dans les suivantes, la $14^{\mathrm{e}}$ (2007), la 15 (2011) et la 16 ${ }^{\mathrm{e}}$ (Grevisse ; Goosse, 2016).

Chargée par la famille de Maurice Grevisse d'actualiser le Français correct, je veillai à introduire les graphies nouvelles dans la $5^{\mathrm{e}}$ édition (1998) de ce Guide pratique, allant jusqu'à donner, dans l'index, la première place à l'entrée en orthographe rectifiée, laissant la seconde à la graphie traditionnelle. La $6^{\mathrm{e}}$ édition les signale également (Lenoble-Pinson, 2009). En matière de langue française, Le bon usage et Le français correct sont des ouvrages de référence, connus et reconnus dans la francophonie et au-delà. 


\subsection{L'Association Pour l'Application des Recommandations Ortho- graphiques ou APARO}

En Belgique, individuellement d'abord, des particuliers (enseignants, amoureux de la langue) adoptèrent les nouvelles manières d'écrire, considérant qu'en ce domaine aucune loi n'avait à leur donner l'autorisation. Certains prirent l'habitude de terminer leur courrier, leurs articles et autres textes par un cachet « Orthographe nouvelle », mettant en évidence l'orthographe utilisée. Fin 1990, quelques-uns d'entre eux se groupèrent autour d'André Goosse pour créer l'Association Pour l'Application des Recommandations Orthographiques (APARO). Aux professeurs se joignirent des hommes politiques, des médecins, des informaticiens, des journalistes et des secrétaires. Cet organisme indépendant de tout pouvoir, pluraliste dans son recrutement, se fit le diffuseur efficace et bénévole des rectifications. Les séances d'information et les conférences, données par ses membres et suivies de débats, éclairèrent le public de manière objective. L'APARO intervint auprès des autorités compétentes pour que les recommandations soient appliquées dans les documents officiels et soient admises dans les épreuves et les examens.

L'APARO élabora et publia deux outils de référence. Le Vadémécum de la nouvelle orthographe contient le relevé complet des mots touchés par les rectifications (APARO, 1995). L'essentiel de la nouvelle orthographe présente la sélection des huit-cents mots les plus fréquents (APARO, 2001). L'un et l'autre expliquent les règles nouvelles, accompagnées de considérations générales qui répondent à d'éventuelles objections. Les deux livrets, tirés à des milliers d'exemplaires, connurent un grand succès, notamment dans le milieu scolaire. L'APARO autorisa leur reproduction en complément des directives données par les responsables des réseaux d'enseignement (voir infra). Les livrets eurent même une certaine diffusion dans quelques pays francophones. Ces listes restent disponibles sur le site du RENOUVO : www.orthographe-recommandee.info. L'APARO publia aussi La Lettre de l'Aparo, un bulletin trimestriel.

\subsection{La presse belge}

Alors qu'en France, les rectifications proposées en 1990 suscitèrent une opposition bruyante, peu attentive au texte du rapport officiel et plutôt faible en qualité d'arguments, les médias belges furent plus placides. Les Belges, il est vrai, sont habitués à la variation linguistique, qu'elle soit due soit à l'influence du néerlandais (une des trois langues nationales), soit à des différences régionales ou sous-régionales. Dans la presse, les échos favorables et défavorables s'équilibrèrent.

Dès 1991, deux mensuels adoptèrent la « nouvelle » orthographe : la Revue générale (fondée en 1865), destinée aux intellectuels, et Diagnostic, revue qui s'adresse aux fonctionnaires de l'administration. Selon une enquête, un tiers des lecteurs de la Revue générale ne remarquait pas les variantes orthographiques. Suivirent le mouvement et appliquèrent les rectifications d'autres périodiques aux contenus divers : intérêt général, bulletins universitaires, études littéraires, revues d'enseignants, illustrés pédagogiques. Un mensuel soigné (Le Lion), envoyé aux consommateurs réguliers d'une société de grande distribution alimentaire, adopta la totalité des rectifications, ce qui entraina l'inquiétude de quelques lecteurs. Ainsi le mot ognon, fré- 
quent dans les recettes de cuisine, suscita-t-il des réactions épistolaires. Une réponse aimable et circonstanciée fut adressée à ces lecteurs attentifs. Le magazine parut de janvier 2003 à janvier 2010 (Éditions Delhaize Le Lion).

Quoique des journalistes se disent favorables, les quotidiens belges n'emploient pas les nouvelles graphies par crainte de perdre des lecteurs. Cependant, en 2008, à la suite de circulaires ministérielles, de la publication des Sept règles pour nous simplifier l'orthographe (Sept règles, 2008) et de graphies rectifiées entrées dans les dictionnaires, la presse emboite le pas et publie des articles d'information. Les sites internet de ces journaux proposent à l'usager un logiciel de conversion, mis au point par le Centre de Traitement Automatique du Langage (CENTAL) à l'Université catholique de Louvain. Le bouton magique appelé « RECTO/VERSO » (RECTifications Orthographiques/VERSion Originale) lui permet de basculer automatiquement de la version traditionnelle d'un texte à la version « rectifiée » ou moderne. Le logiciel convertit à la demande n'importe quel texte en nouvelle orthographe et souligne les mots modifiés; de plus, si l'usager glisse le curseur sur un mot souligné, il fait apparaitre une bulle contenant l'explication de la modification. Chaque semaine, un million de textes passent ainsi à la nouvelle orthographe.

\subsection{L'enseignement primaire, secondaire et universitaire}

Dans les départements de philologie et de langues romanes des universités, les rectifications sont enseignées et leur emploi est recommandé. Des professeurs les appliquent dans leurs notes de cours comme dans leurs publications (Lenoble-Pinson, 2019). On les emploie dans beaucoup de hautes écoles, qui forment les instituteurs et les maitres habilités à enseigner dans le degré inférieur du secondaire.

L'APARO intervint à plusieurs reprises auprès des responsables de l'enseignement pour que l'information relative aux rectifications soit donnée aux enseignants et que les graphies nouvelles ne soient pas considérées comme fautives, ainsi que le recommande l'Académie française. Ces interventions donnèrent de bons résultats dans l'enseignement catholique, important quant au nombre d'élèves, où la nouvelle orthographe fut bien accueillie. Plusieurs programmes alors écrits en nouvelle orthographe en recommandent l'usage. Dans l'enseignement d'État, ce fut plus lent. En mars et en aout 1998, deux circulaires d'information, adressées par le ministère de l'Éducation aux directeurs des établissements d'enseignement supérieur (universités et hautes écoles) et aux directeurs de toutes les écoles fondamentales, secondaires et spéciales, les informèrent de l'existence de la nouvelle orthographe. Ces Recommandations relatives à l'application de la nouvelle orthographe proposèrent aux enseignants d'acquérir gratuitement le livret de l'APARO, L'Essentiel de la nouvelle orthographe (APARO, 2001).

Le contenu des circulaires est explicite. «Il n'est certainement pas recommandé d'imposer une, et une seule orthographe. Chacun a le droit d'utiliser les différentes graphies. Il s'ensuit que, durant une période de durée indéterminée, les deux orthographes auront à coexister et seront acceptées. En conséquence, lors des contrôles, les deux orthographes seront admises. [...] Les rectifications apportées obéissent à des règles générales souffrant peu d'exceptions. Aussi les élèves et les étudiants abordant l'apprentissage de l'orthographe verront-ils leur tra- 
vail simplifié et leurs performances améliorées. Il s'indique donc, avec eux, afin d'éviter toute confusion, de ne plus enseigner de règles opposées à la nouvelle orthographe. [...] Il conviendra d'informer le plus largement possible tous les membres de la communauté éducative de ces dispositions. Ceux-ci veilleront à se tenir informés des recommandations orthographiques. » Cette initiative du ministère de l'Éducation mit fin aux bruits divers qui circulaient dans les écoles à propos de la légitimité de la nouvelle orthographe. Leur enseignement s'installa dans de nombreuses écoles primaires, secondaires et supérieures.

Confortés par la déclaration de l'Académie française dans sa séance du 17 janvier 1991, par les documents ministériels belges, par les interventions écrites et publiques d'André Goosse, les auteurs de manuels scolaires ne craignent pas d'adopter les rectifications (Dupriez, 2018 et 2013). Ils signalent leur option par une phrase liminaire du type « Ce livre applique les recommandations orthographiques de 1990 ». Cette mention tend à disparaitre.

\subsection{La Société belge des professeurs de français (SBPF) devenue l'Association belge des professeurs de français (ABPF)}

Pendant plusieurs années, la SBPF admit les rectifications dans sa revue Français 2000 tout en laissant les auteurs libres d'employer les graphies nouvelles ou traditionnelles. À la suite d'une enquête, elle consacra le numéro de septembre 2009 à la nouvelle orthographe, qu'elle adopta entièrement, y compris sur son site : www.abpf.be.

Le site de l'enseignement de la Fédération Wallonie-Bruxelles suit les recommandations : www.enseignement.be, y compris dans la revue téléchargeable Prof, depuis juin 2009. L’astérisque qui signalait les formes rectifiées est abandonné en juin 2012. - Depuis janvier 1991, le mouvement Freinet les utilise dans ses nombreuses publications.

\subsection{La Maison de la Francité}

Depuis 1997, la Maison de la Francité, à Bruxelles, contribue à la diffusion de la réforme orthographique. Elle accueillit en ses murs un colloque-bilan de l'APARO réunissant des linguistes suisses, québécois, français et belges. Elle organisa des conférences en s'engageant du côté des réformateurs. En octobre 2001, une de ses priorités étant de «moderniser la langue française », la Maison confirma son appui aux « réformes visant à la simplification de l'orthographe ». Les illogismes, exceptions et bizarreries héritées du passé gênent gravement l'apprentissage du français écrit, à fortiori quand il n'est pas la langue maternelle du locuteur. En 2002, en concertation avec l'APARO, la Maison de la Francité mena une enquête sur l'application de la réforme et sur ses obstacles, dans les 252 écoles primaires francophones de la Région bruxelloise. En avril 2004, elle invita les instituteurs bruxellois à une séance d'information et diffusa les livrets de l'APARO et du RENOUVO. Elle poursuit dans cette voie. Entretemps, la revue Francité s'écrit en nouvelle orthographe ainsi que les textes lauréats publiés après un concours annuel. - www.maisondelafrancite.be 


\section{En Francophonie}

\subsection{Le RENOUVO}

Avec la volonté de diffuser les rectifications, les responsables de trois associations, sises dans trois pays voisins, fondent le RENOUVO, le REseau pour la NOUVelle Orthographe du français. Unissent leurs forces : en France, l'AIROÉ (Association pour l'Information et la Recherche sur les Orthographes et les systèmes d'Écriture), en Suisse, l'ANO (Association pour la Nouvelle Orthographe), et en Belgique, l'APARO (Association Pour l'Application des Recommandations Orthographiques), déjà présentée. Le RENOUVO s'élargira au Québec, en incluant le GQMNF (Groupe Québécois pour la Modernisation de la Norme du Français), et à Haïti. La petite équipe européenne, dynamique, se réunit à Paris et à Bruxelles. Elle prépare et publie, en 2002, Le millepatte sur un nénufar, livret illustré contenant les règles nouvelles et la liste alphabétique des rectifications. Sa typographie est significative : les formes de haute fréquence sont écrites en caractères gras (gout n.m.) ; les formes de fréquence moyenne, en caractères standards (gouteux, euse adj.) ; les formes de basse fréquence sont données dans un corps plus petit (gouteur, euse n.). Le livret plait, notamment dans les classes. Il doit être réédité plusieurs fois (RENOUVO, 2005).

L'action du RENOUVO se poursuit sur deux sites libres d'accès, tenus à jour par un membre suisse (Ramat ; Muller, 2009) : www.renouvo.org et www.orthographe-recommandee.info. Le site www.renouvo.org donne les positions officielles, les consignes ministérielles pour l'enseignement, les nouvelles règles, des exercices en ligne, les dictionnaires et les guides de conjugaison, des lectures diverses en nouvelle orthographe. Le site www.orthographe-recommandee. info, destiné aux professionnels comme au grand public, fournit les informations relatives aux logiciels de correction, un miniguide téléchargeable contenant les modifications, la liste alphabétique des mots touchés, les documents des ministères de l'Éducation, etc.

Afin de promouvoir l'orthographe recommandée ont été créés un label de qualité spécifique et des logos de conformité « Orthographe recommandée ». Décernés aux livres et aux produits informatiques incluant les rectifications, ils garantissent une intégration parfaite de la nouvelle orthographe. Le label a déjà été refusé à certains produits.

\subsection{Les Championnats d'orthographe de Belgique}

Voir le site https : www.championnats-orthographe.be

Les passionnés qui participent aux Championnats d'orthographe de Belgique considèrent la dictée comme un sport cérébral et un plaisir. Fondés en 1971, les Championnats servirent de modèle à ceux de Bernard Pivot, en France. Leur particularité est d'emprunter les textes des dictées aux écrivains belges et de contribuer ainsi à la diffusion de la littérature française écrite en Belgique, entre autres par Henry Bauchau, Michel de Ghelderode ou Georges Simenon. Les difficultés des dictées sont maitrisables par des élèves de classe terminale de l'enseignement secondaire. À la demande d'un fidèle candidat, les rectifications sont admises dans les dictées depuis 1991. Il ne s'agit pas d'un concours. Tous les candidats qui écrivent une dictée sans faute deviennent lauréats et obtiennent le prestigieuse Carte de lauréat des Championnats d'orthographe ainsi qu'un logiciel Cordial, correcteur d'orthographe et de grammaire. La Carte de 
lauréat, qui constitue un brevet de connaissance pratique de l'orthographe, est très appréciée sur le marché de l'emploi.

Des recueils intitulés Écrire sans faute réunissent les dictées des Championnats d'orthographe accompagnées de commentaires lexicaux et grammaticaux, écrits en orthographe recommandée. La liste alphabétique des huit-cents mots rectifiés les plus fréquents termine chaque livre (Lenoble-Pinson, 2017).

\subsection{La Dictée des Amériques}

Pendant quinze ans, de 1995 à 2009, trois lauréats belges des Championnats d'orthographe de l'année participèrent au Québec à la finale internationale de la Dictée des Amériques. Sur ces quarante-cinq finalistes, seize obtinrent le titre de «Champion » ou de «Grand Champion » des Amériques » ! Chargée de la présidence de la demi-finale belge, j’organisais chaque année les épreuves à Bruxelles en admettant les rectifications de 1990.

\subsection{Les correcteurs informatiques}

Les logiciels de correction sont largement utilisés, y compris dans l'enseignement, où leur emploi raisonné peut être recommandé par les programmes scolaires. Ils sont efficaces en matière d'orthographe lexicale et apportent une aide à la rédaction en nouvelle orthographe. Aujourd'hui, tous les correcteurs informatiques, vérificateurs courants ou avancés, tiennent compte des rectifications. Dans la pratique, l'utilisateur a le choix, au moins, entre une correction en nouvelle orthographe et une correction en orthographe traditionnelle.

Les correcteurs se classent en deux catégories. D'une part, les vérificateurs courants, intégrés dans des logiciels comme Word, OpenOffice.org, Outlook ou Firefox, servent surtout à corriger l'orthographe lexicale. Ce sont des accessoires pratiques mais à efficacité réduite. D'autre part, les correcteurs avancés, plus performants, s'intègrent aux logiciels de traitement de texte mais doivent être achetés séparément. Leur prix varie à partir de cent euros. Les plus connus sont Antidote chez Druide informatique (www.druide.com) ; Cordial chez Synapse Développement (www.synapse-developpement.fr) ; et ProLexis aux éditions Diagonal. Ils analysent les phrases et vérifient l'orthographe lexicale ainsi que les accords, l'emploi des modes, le respect des règles typographiques, etc. Ils sont dotés de fonctions supplémentaires et de réglages personnalisables (comme : tolérer les majuscules non accentuées).

Le correcteur Antidote (www.antidote.info) est accompagné d'un dictionnaire de définitions, d'un dictionnaire de synonymes, d'un conjugueur et d'un mémento grammatical de huit-cents articles clairs et précis. Le correcteur français Cordial offre également des dictionnaires complémentaires. Le correcteur ProLexis, plus rapide, moins pédagogique, est utilisé par les professionnels (journalistes, éditeurs, institutions de l'Union européenne). Les correcteurs orthographiques favorisent la propagation des graphies réformées dans l'usage et chez les scripteurs, Antidote décrivant en détail chaque règle nouvelle dans sa grammaire.

\section{Et demain ?}

Existe-t-il des projets de rationalisation de l'orthographe? Oui. 


\subsection{Des réformes ponctuelles}

Des projets de réformes ponctuelles, limitées et raisonnables, pourraient faire évoluer l'orthographe d'usage vers plus de rationalisation. Le groupe de travail ÉROFA (Études pour une Rationalisation de l'Orthographe Française d'Aujourd'hui), sous la direction de Claude Gruaz, a publié, en orthographe rectifiée, des Études pour une rationalisation de l'orthographe française (Gruaz, 2009-2018). Les études, très intéressantes, sur les consonnes doubles, le $x$ final et les lettres grecques se terminent chacune par une proposition de réforme. Par exemple, en finale de mot, le $x$ non prononcé pourrait être remplacé par $s$ : des cheveus, des agneaus. Les graphèmes grecs et similaires pourraient laisser leur place aux graphèmes français correspondants : farmacie comme fantasme. En 1820, le père d'Ambroise Firmin-Didot écrivait dans une note : « au moment de quitter la carrière typographique, je suis las de feuilleter sans cesse des dictionnaires qui se contredisent entre eux et se contredisent eux-mêmes. [...] je voudrais qu'on écrivît le mot philosophe non-seulement avec un $f$ à la dernière syllabe, comme le proposait de Wailly, mais je mettrais ce $f$ même à la première syllabe, comme font les Italiens et les Espagnols. » L'orthographe, « cessons de la considérer comme tabou : elle n'est que la servante du langage et non pas sa maitresse » (Hervé Bazin). Si l'on ne change rien, l'orthographe ne changera pas.

\subsection{Une rationalisation de l'accord du participe passé}

$\mathrm{Au}$ XVIII ${ }^{\mathrm{e}}$ siècle, le père Buffier, dans sa Grammaire françoise, estime qu'« on pourroit sans comettre de faute rendre toujours le participe indéclinable ». Rationaliser l'orthographe française, c'est libérer l'écrit et le mettre à la portée de la plupart des citoyens. Les recommandations relatives aux accords du participe passé sont accessibles en ligne sur le site http://participepasse.info. Leur application est plus avancée que celle des réformes ponctuelles évoquées ci-dessus.

Les participes passés conjugués avec l'auxiliaire avoir sont invariables, comme c'est fréquemment le cas dans l'usage oral. La lettre que j'ai écrit comme j'ai écrit la lettre. «Écoutez-les : les plus grandes personnalités de ce pays, Charles de Gaulle lui-même, la plupart des hommes politiques, des comédiens, professeurs, avocats, académiciens, tous ceux qui ont le pouvoir de la parole, ne font plus l'accord à l'oral qu'une fois sur trois ou quatre, et ils ont bien raison », écrit Nina Catach en 1989. En français, l'écart entre l'oral et l'écrit s'est creusé et l'on n'a pas procédé aux réajustements qui s'imposaient. La non-réalisation de cet accord du participe passé peut être considérée comme une régulation naturelle. Le premier livre à appliquer délibérément l'invariabilité du participe passé conjugué avec l'auxiliaire avoir paraitra en septembre 2020 aux éditions Le Robert, avec le logo « Orthographe recommandée » du RENOUVO (voir supra). Sous le titre Le français n'existe pas, il rassemblera les chroniques « Tu parles ! » d'Arnaud Hoedt et de Jérôme Piron, diffusées sur France inter pendant l'été 2019 (Hoedt ; Piron, 2020). Comme pour les rectifications de 1990, les règles traditionnelles restent valables. Les deux types d'accord ont à coexister dans l'usage, oral et écrit, comme ils coexistent déjà, et depuis longtemps.

\subsection{Le Conseil international de la langue française (CILF, Paris)}

Voir le site à www.cilf.fr - cilf@cilf.fr 
Le CILF a pour tâche d'enrichir la langue française et de favoriser son rayonnement dans la francophonie tout en organisant le dialogue avec les autres langues. Le CILF s'occupe notamment de terminologie. Il a publié une centaine de dictionnaires spécialisés mono- et multilingues, sur papier et sur l'internet, dont un dictionnaire de médecine (54 000 termes) et un dictionnaire du génie civil (8 000 termes). Il propose une base de terminologie scientifique et technique de 120000 termes. Le CILF traite aussi d'orthographe (Dister et al., 2009), de grammaire et de linguistique. Depuis 1991, il emploie les rectifications dans ses publications et appuie les projets de rationalisation de l'orthographe. Il gère ORTHONET, une banque de données orthographiques et grammaticales de 25000 termes, comprenant les réponses aux questions les plus fréquentes et des jeux - http://orthonet.sdv.fr/. Il édite Le français moderne, une revue de linguistique (fondée en 1933).

Qu'il s'agisse des rectifications de 1990 ou des règles de rationalisation de l'accord du participe passé, le Conseil international de la langue française ainsi que la Fédération internationale des professeurs de français (F.I.P.F.) et l'Association belge des professeurs de français (A.B.P.F.) en recommandent vivement l'emploi à tous les enseignants.

\section{Conclusion}

L'orthographe nouvelle se répand sans être enseignée. De plus en plus de scripteurs l'utilisent spontanément sans l'avoir apprise, elle est donc quotidiennement mêlée à l'orthographe traditionnelle. Puisqu'elle va dans le sens d'une rationalisation du système, on eût pu souhaiter une mise en ordre plus ample. Pourtant, d'aucuns s'interrogent encore (Legros ; Moreau, 2012).

Les rectifications de 1990 constituent-elles une révolution? Non. Il est bon de rappeler qu'en France, plus d'un mot sur deux a changé au moins une fois d'orthographe depuis le XVI ${ }^{\mathrm{e}}$ siècle, et parfois à plusieurs reprises. Les quelque 2000 graphies plus ou moins modifiées en 1990 ne représentent pas une révolution, « ni même une vraie réforme, mais quelques améliorations comme celles que l'Académie a apportées à chaque édition de son dictionnaire, en provoquant les mêmes gémissements : poëte a perdu sa poésie avec son tréma, phthisie son agressivité avec son deuxième $h$, disait-on en 1878 » (Goosse, 1995). Les rectifications correspondent en grande partie à l'évolution naturelle de la prononciation et des graphies, ce qui rend l'apprentissage de l'orthographe plus aisé et surtout plus cohérent.

Les graphies rectifiées gênent-elles la lecture ? Non. Des enquêtes montrent que la plupart des lecteurs ne les voient pas. Elles sont d'ailleurs peu nombreuses et donc peu visibles. Lorsqu'on les applique toutes, seul un mot toutes les deux pages est modifié, et souvent il ne s'agit que d'un accent. Donc, les rectifications ne gênent pas la lecture.

Les graphies rectifiées peuvent-elles cohabiter avec les anciennes ? Oui. Dans l'écriture, l'orthographe reste la règle. Le laxisme est socialement pénalisant alors qu'un « espace de liberté », défendu par Robert Martin, membre de l'Institut, peut être admis. Cet « espace de variation minimale » permet de procéder à des aménagements correspondant à l'évolution de l'usage. Les variantes orthographiques que sont les rectifications trouvent leur place dans cet espace de liberté. Elles ne sont pas imposées, mais sont recommandées. Aux 3000 variantes 
anciennes, du type clef ou clé, il payera ou paiera, s'ajoutent désormais 2000 variantes « nouvelles » (elles ont trente ans !). L'orthographe française compte désormais 5000 variantes. Aucune des deux graphies (ni la nouvelle ni l'ancienne) ne peut être tenue pour fautive, précise l'Académie française au début de chaque fascicule de la neuvième édition de son Dictionnaire (Dictionnaire, 1992-...).

Les deux graphies des mots modifiés resteront admises jusqu'à ce que la nouvelle soit entrée dans l'usage. Durant son histoire, l'Académie française n’a jamais renoncé à une réforme graphique qu'elle avait proposée. Néanmoins, les graphies anciennes restent valables. En pensant aux usagers, il importe de ne pas considérer comme faute ce qui est logique et donc de ne pas pénaliser les graphies nouvelles qui, en conséquence, disposeront du temps nécessaire pour s'implanter. Pendant un temps indéterminé, les deux orthographes auront à coexister. L'inquiétude n'est pas de mise. La langue n'est pas touchée. Seul son vêtement s'adapte à l'usage de notre époque. Les correcteurs orthographiques - comme Antidote et Cordial - et les dictionnaires - comme le Dictionnaire Hachette (depuis 2005) et le Petit Larousse illustré (depuis 2012) - confortent l'implantation des nouvelles graphies puisqu'ils les signalent toutes, ce qui peut rassurer les inquiets.

«Supprimer quelques anomalies, entériner dans l'orthographe des changements de la prononciation, introduire des règles là où règnent l'arbitraire et le désordre, c'est rendre un service modeste mais réel aux usagers, surtout aux usagers futurs ; c'est servir, modestement je le répète, la cause du français dans le monde. » (Goosse, 1995 : 8).

\section{Bibliographie}

\ APARO (Association Pour l'Application des Recommandations Orthographiques) (2001) [1997]. L'essentiel de la nouvelle orthographe. Les huit-cents mots les plus fréquents. Abrégé du vadémécum. Bruxelles : Aparo ( $4^{e}$ édition).

У APARO (1995) [1992]. Vadémécum de la nouvelle orthographe. Bruxelles : Aparo ( ${ }^{2}$ édition).

$\checkmark$ BIEDERMANN-PASQUES, Liselotte ; JEJCIC, Fabrice (éds) (2006). Les rectifications orthographiques de 1990. Analyses des pratiques réelles (Belgique, France, Québec, Suisse, 2002-2004). Université d'Orléans : Presses universitaires d'Orléans.

У Dictionnaire de l'Académie française, 1992-... [1694]. Paris, Imprimerie nationale - Librairie Arthème Fayard, 3 tomes parus, jusqu'à quotité; Documents administratifs du Journal officiel de la République française, fascicules parus jusqu'à sérénissime, 19 février 2020 ( $9^{\mathrm{e}}$ édition).

У DISTER, Anne; GRUAZ, Claude ; LEGROS, Georges ; LENOBLE-PINSON, Michèle ; MOREAU, Marie-Louise ; Petit, Christine ; VAn Raemdonck, Dan ; wilmet, Marc (2009). Penser l'orthographe de demain. Paris : Conseil international de la langue française.

У DUPRIEZ, Dominique (2013). La nouvelle orthographe en pratique. Tome 2 : Exercices et approches pédagogiques. Bruxelles : De Boeck Supérieur.

У DUPRIEZ, Dominique (2018). La nouvelle orthographe en pratique. Louvain-la-Neuve : De Boeck Supérieur ( $3^{\mathrm{e}}$ édition).

У Goosse, André (1991). La «nouvelle » orthographe. Exposé et commentaires. Paris / Louvain-la-Neuve : Éditions Duculot. 
У GOOSSE, André (1995). «Servir la cause du français dans le monde », La libre Belgique, 14-15 janvier $1995: 8$.

У GREVISSE, Maurice; Goosse, André (2016) [1936]. Le bon usage. Louvain-la-Neuve: De Boeck Supérieur (16 édition).

У GRUAZ, Claude (dir.) (2009-2018). Études pour une Rationalisation de l'Orthographe Française d'Aujourd'hui (ÉROFA). Limoges : Éditions Lambert-Lucas, coll. « Le débat orthographique ».

$\rightarrow$ (2009). Le $\mathrm{x}$ final (1 600 mots).

$\rightarrow$ (2009). Les consonnes doubles. Féminins et dérivés (2 050 mots).

$\rightarrow \quad$ (2010). Les consonnes doubles après -e (3 500 mots).

$\rightarrow$ (2012). L'accord du participe passé.

$\rightarrow$ (2013). Simplifier les consonnes doubles (7500 mots).

$\rightarrow$ (2015). Les lettres grecques et similaires (6 250 entrées).

$\rightarrow$ (2018). Dictionnaire de l'orthographe rationalisée du français (14 500 mots).

У HOEDT, Arnaud; PIRON, Jérôme (2020). Le français n'existe pas. Paris : Le Robert.

\ LEGROS, Georges ; MOREAU, Marie-Louise (2012). Orthographe : qui a peur de la réforme? Bruxelles : Ministère de la Fédération Wallonie-Bruxelles.

$\checkmark$ LENOble-PInson, Michèle (dir.) (2005) [1994]. Mettre au féminin. Guide de féminisation des noms de métier, fonction, grade ou titre. Bruxelles : Service de la langue française et Conseil supérieur de la langue française (2e édition).

У LENOBLE-PINSON, Michèle (2009) [1998]. Le français correct. Guide pratique des difficultés de M. Grevisse. Bruxelles : Groupe De Boeck (6e édition).

y LenOble-PINSON, Michèle (2017) [2005]. Écrire sans faute. [33] Dictées [des Championnats d'orthographe] lues, commentées et corrigées. Louvain-la-Neuve, De Boeck Supérieur ( $3^{\mathrm{e}}$ édition) : Textes audio téléchargeables.

ע LENOBLE-PINSON, Michèle (2019) [2014]. Dire et écrire le droit en français correct. Au plaisir des gens de robe. Bruxelles : Éditions Bruylant (2 édition).

$\checkmark$ RAMAT, Aurel; MULLER, Romain. (2009). Le Ramat européen de la typographie. Dijon : Éditions De Champlain.

У RENOUvo (Réseau pour la Nouvelle Orthographe du français) (2005) [2002]. Le millepatte sur un nénufar. Vadémécum de l'orthographe recommandée, France-Suisse-Belgique, AIROÉ-ANO-APARO (3 édition).

\ Sept règles pour nous simplifier l'orthographe (2008). Bruxelles : Ministère de la Communauté française.

\author{
Michèle Lenoble-Pinson \\ «Le Clos joyeux » \\ Avenue Winston Churchill, 165 D, Bte 62 \\ 1180 BRUXELLES \\ Belgique
}

